\title{
Recent advances in electron cryotomography and their application to imaging purified protein complexes, isolated organelles, and small cells.
}

\author{
Cristina V. Iancu, Gavin E. Murphy, Elizabeth R. Wright, Jordan Benjamin, \\ William F. Tivol, D. Prabha Dias, J. Bernard Heymann, and Grant Jensen
}

Biology Division, California Institute of Technology, Pasadena, California, 91125

A new 300kV, FEG, helium-cooled, energy-filtered "G2 Polara" electron cryoTEM was recently installed at Caltech. Using this instrument, we have now extensively tested the potential advantages of liquid helium cooling and the inclusion of two orthogonal tilt-series in the context of electron cryotomography. These results and the application of the technology to several specific biological problems are reported.

The first cryostages used liquid nitrogen to keep samples frozen. After it was observed that cooled samples diffracted longer under irradiation than room-temperature samples, it was hoped that further cooling might result in additional radiation protection. New stages were built that could accommodate liquid helium as the cryogen. Disappointingly, we have now found that liquid helium cooling is actually disadvantageous, at least for the doses and resolutions of interest in electron tomography, because the contrast from proteins and lipid bilayers gradually fades. In an attempt to explain this observation, we have explored the nature of vitreous ice when irradiated at $\sim 82 \mathrm{~K}, \sim 12 \mathrm{~K}$, and when warmed or cooled between the two temperatures. Specifically, we have recorded electron diffraction patterns, burned "tunnels" through the ice to measure thickness directly, and tracked the positions of gold fiducials within the ice at the two temperatures and through transitions. Confirming existing literature, these studies show that vitreous ice condenses from a lower to a higher density state when irradiated with even 2 electron $/ \AA^{2}$ at $\sim 12 \mathrm{~K}$, and that this transition slowly but spontaneously reverts when the sample is subsequently warmed. Again in agreement with existing literature, we have seen that the higher density state is less viscous. In addition, we have observed that certain strains that build up at $\sim 12 \mathrm{~K}$ are relieved by warming. We hypothesize that the loss-of-contrast effects that make helium cooling undesirable are not caused principally by the density change itself, but instead by the reduced mobility of radiolytic fragments in the high density state.

The new microscope has made the collection of dual-axis tilt-series of cryosamples routine, and we have now collected more than a hundred such data sets from various samples. These demonstrate that inclusion of the second tilt-series results in more isotropic resolution as expected, clarifying the morphology of membranes, protein shells, and individual protein complexes. Sometimes dual-axis tilting has proven essential, including one case in which a key bacterial cytoskeletal filament was practically invisible in the tomogram from the first tilt-series, but was clearly resolved in the second. Indeed long, tubular features that lie perpendicular to the tilt-axis are nearly invisible to single-axis tomography. Nevertheless, our efforts have been the 
first attempts to our knowledge to merge dual-axis tilt-series of isolated macromolecules suspended in vitreous ice, and two problems emerged which demand the development of more sophisticated software for aligning and merging such data. First, methods to locally align objects in the two tilt-series before merging are needed which work well for small, isolated particles within a relatively featureless background of vitreous ice. Second, better algorithms are needed to properly scale the merged amplitudes of each region in reciprocal space.

Using this new instrumentation we have recorded three-dimensional reconstructions of several purified protein complexes, isolated organelles, and small cells. In one example, dual-axis tiltseries of purified pyruvate dehydrogenase multienzyme complex (PDMC) from E. coli were recorded. PDMC is a 5.6 MDa complex consisting of three major proteins referred to as E1, E2, and E3. Its octahedral core is formed of 24 copies of the E2 enzyme, where each "corner" is composed of an E2 trimer. The N-terminal portion of E2 is a presumably disordered polypeptide that contains three lipoyl domains that bind the substrate and shuttle it between the various active sites, and a fourth domain capable of binding either E1 or E3. X-ray crystal or NMR structures are available for every piece that is expected to be structured, but how these pieces are arranged in the full complex is unknown. Our results show that the E1 and E3 domains are not specifically bound to the $\mathrm{E} 2$ core, but rather are flexibly tethered. In addition, each of the $80 \mathrm{kDa}$ corners of the E2 core could be clearly distinguished, setting a new standard in our ability to visualize quaternary structure.

As another example, we have recorded three-dimensional reconstructions of carboxysomes from the prokaryote Synechococcus species. Carboxysomes are $\sim 100 \mathrm{~nm}$ microcompartments consisting of a proteinaceous shell surrounding many tens of copies of the enzyme ribulose1,5bisphosphate carboxylase/oxygenase (RuBisCO), as well as other, smaller enzymes. The shape of the protein shell and many individual RuBisCO molecules were resolved in the reconstructions. 